\title{
On the Generalized Hyers-Ulam Stability of an $n$-Dimensional Quadratic and Additive Type Functional Equation
}

\author{
Yang-Hi Lee \\ Department of Mathematics Education, Gongju National University of Education, Gongju 314-711, Republic of Korea \\ Correspondence should be addressed to Yang-Hi Lee; yanghi2@hanmail.net
}

Received 13 February 2014; Accepted 28 April 2014; Published 26 May 2014

Academic Editor: Sabri Arik

Copyright (c) 2014 Yang-Hi Lee. This is an open access article distributed under the Creative Commons Attribution License, which permits unrestricted use, distribution, and reproduction in any medium, provided the original work is properly cited.

We investigate the generalized Hyers-Ulam stability of a functional equation $f\left(\sum_{j=1}^{n} x_{j}\right)+(n-2) \sum_{j=1}^{n} f\left(x_{j}\right)-\sum_{1 \leq i<j \leq n} f\left(x_{i}+x_{j}\right)=0$.

\section{Introduction}

Throughout this paper, let $X$ be a normed space and $Y$ a Banach space. For a given mapping $f: X \rightarrow Y$, we define

$$
\begin{gathered}
A f(x, y):=f(x+y)-f(x)-f(y), \\
Q f(x, y):=f(x+y)+f(x-y)-2 f(x)-2 f(y),
\end{gathered}
$$

for all $x, y \in X$. A mapping $f: X \rightarrow Y$ is called an additive mapping (a quadratic mapping, resp.) if $f$ satisfies the functional equation $A f=0(Q f=0$, resp.). If a mapping is represented by sum of an additive mapping and a quadratic mapping, we call the mapping a quadratic-additive mapping. For a functional equation $E f=0$ if all of the solutions of $E f=0$ are quadratic-additive mappings and all of quadraticadditive mappings are the solutions of $E f=0$, then we call the functional equation $E f=0$ a quadratic-additive type functional equation.

In 1940, Ulam [1] raised a question concerning the stability of homomorphisms. Hyers [2], Aoki [3], Rassias [4], and Găvruţa [5] made important role to study the stability of the functional equation. During the last decades, the stability problems of functional equations have been extensively investigated by a number of mathematicians (see also [6-9]).

In 2006, Jun and Kim [10] obtained the stability of the functional equation

$$
\begin{array}{r}
f\left(\sum_{j=1}^{n} x_{j}\right)+(n-2) \sum_{j=1}^{n} f\left(x_{j}\right) \\
-\sum_{1 \leq i<j \leq n} f\left(x_{i}+x_{j}\right)=0,
\end{array}
$$

for all $x_{1}, x_{2}, \ldots, x_{n} \in X(n>2)$ (see also [11-15]). The functional equation (2) is a quadratic-additive type functional equation (see Theorem 2.6 in [16]). For the case $n=3$, Jung [17] proved the stability of the functional equation (2) (see also [18-20]) and, for the case $n=4$, Chang et al. [21] proved the stability of the functional equation (2) (see also [22-25]).

In this paper, we will generalize the previous results of the stability problem of the functional equation (2) on the punctured domain. In particular, we will show the superstability (if $p<0$ ) of the functional equation (2) in the sense of Rassias. 


\section{Stability of the Functional Equation (2) ( $n$ Is Even)}

Let $(s, t)$ be a fixed element in $\{(1,1),(1,-1),(-1,-1)\}$ and let $\varphi:(X \backslash\{0\})^{n} \rightarrow[0, \infty)$ be a function satisfying the conditions:

$$
\begin{aligned}
& \sum_{j=0}^{\infty} 4^{-s j} \varphi\left(2^{s j} x_{1}, 2^{s j} x_{2}, \ldots, 2^{s j} x_{n}\right)<\infty \\
& \sum_{j=0}^{\infty} 2^{-t j} \varphi\left(2^{t j} x_{1}, 2^{t j} x_{2}, \ldots, 2^{t j} x_{n}\right)<\infty
\end{aligned}
$$

for all $x_{1}, x_{2}, \ldots, x_{n} \in X \backslash\{0\}$, where $n$ is a fixed even integer greater than 2 in this section. For convenience, we use the following abbreviations in this section for a given mapping $f: X \rightarrow Y:$

$$
\begin{aligned}
& \operatorname{Df}\left(x_{1}, x_{2}, \ldots, x_{n}\right) \\
& :=f\left(\sum_{j=1}^{n} x_{j}\right)+(n-2) \sum_{j=1}^{n} f\left(x_{j}\right)-\sum_{1 \leq i<j \leq n} f\left(x_{i}+x_{j}\right), \\
& J_{m} f(x) \\
& =\frac{1}{2}\left(4^{-s m}\left(f\left(2^{s m} x\right)+f\left(-2^{s m} x\right)-\frac{2(n+2)}{3 n} f(0)\right)\right. \\
& \left.+2^{-t m}\left(f\left(2^{t m} x\right)-f\left(-2^{t m} x\right)\right)\right), \\
& \bar{x}:=(\overbrace{x, \ldots, x}^{n / 2+1}, \overbrace{-x, \ldots,-x}^{n / 2-1}),
\end{aligned}
$$

for all $x, x_{1}, x_{2}, \ldots, x_{n} \in X$. From these, we get the equality

$$
\begin{aligned}
J_{m} f(x)-J_{m+1} f(x) \\
=\frac{2 \cdot 4^{\tau_{-s, m}}}{n(n-2)}\left(D f\left(\overline{2^{\tau_{s, m} x}}\right)+D f\left(\overline{-2^{\tau_{s, m} x}}\right)\right) s \\
\quad+\frac{2^{\tau_{-t, m}-1}}{n-2}\left(D f\left(\overline{2^{\tau_{t, m} x}}\right)-D f\left(\overline{-2^{\tau_{t, m} x}}\right)\right) t
\end{aligned}
$$

for all $x \in X \backslash\{0\}$ and all nonnegative integers $m$, where $\tau_{k, m}$ are the integers defined by

$$
\tau_{k, m}=k\left(m+\frac{1}{2}\right)-\frac{1}{2}
$$

for $k \in\{-1,1\}$.

Lemma 1. If $f: X \rightarrow Y$ is a mapping such that

$$
\text { Df }\left(x_{1}, x_{2}, \ldots, x_{n}\right)=0 \text {, }
$$

for all $x_{1}, x_{2}, \ldots, x_{n} \in X \backslash\{0\}$, then

$$
J_{m} f(x)=f(x)-\frac{n+2}{3 n} f(0)
$$

for all $x \in X \backslash\{0\}$ and all nonnegative integers $m$.
Proof. We can easily get

$$
\begin{gathered}
f(x)-\frac{n+2}{3 n} f(0)-J_{m} f(x) \\
=\sum_{j=0}^{m-1}\left(\frac{2 \cdot 4^{\tau_{-s, j}}}{n(n-2)}\left(D f\left(\overline{2^{\tau_{s, j} x}}\right)+D f\left(\overline{-2^{\tau_{s, j} x}}\right)\right) s\right. \\
\left.\quad+\frac{2^{\tau_{-t, j}-1}}{n-2}\left(D f\left(\overline{2^{\tau_{t, j} x}}\right)-D f\left(\overline{-2^{\tau_{t, j} x}}\right)\right) t\right) \\
=0
\end{gathered}
$$

for all $x \in X \backslash\{0\}$ and all nonnegative integers $m$.

Theorem 2. Suppose that $f: X \rightarrow Y$ is a mapping such that

$$
\left\|D f\left(x_{1}, x_{2}, \ldots, x_{n}\right)\right\| \leq \varphi\left(x_{1}, x_{2}, \ldots, x_{n}\right)
$$

for all $x_{1}, x_{2}, \ldots, x_{n} \in X \backslash\{0\}$ with $\lim _{m \rightarrow \infty} J_{m} f(0)=0$. Then, there exists a unique mapping $F: X \rightarrow Y$ satisfying $(8)$ for all $x_{1}, x_{2}, \ldots, x_{n} \in X \backslash\{0\}$ and

$$
\left\|f(x)-\frac{n+2}{3 n} f(0)-F(x)\right\| \leq \sum_{j=0}^{\infty} \Phi_{j}(x)
$$

for all $x \in X \backslash\{0\}$ with $F(0)=0$, where $\Phi_{j}$ are the mappings defined by

$$
\begin{aligned}
\Phi_{j}(x):= & \frac{2 \cdot 4^{\tau_{-s, j}}}{n(n-2)}\left(\varphi\left(\overline{2^{\tau_{s, j} x}}\right)+\varphi\left(\overline{-2^{\tau_{s, j} x}}\right)\right) \\
& +\frac{2^{\tau_{-t, j}-1}}{n-2}\left(\varphi\left(\overline{2^{\tau_{t, j} x}}\right)+\varphi\left(\overline{-2^{\tau_{t, j} x}}\right)\right)
\end{aligned}
$$

for all $x \in X \backslash\{0\}$.

Proof. It follows from (6) and (11) that

$$
\begin{gathered}
\left\|J_{m} f(x)-J_{m+m^{\prime}} f(x)\right\| \\
=\sum_{j=m}^{m+m^{\prime}-1} \| \frac{2 \cdot 4^{\tau_{-s, j}}}{n(n-2)}\left(D f\left(\overline{2^{\tau_{s, j}} x}\right)+D f\left(\overline{-2^{\tau_{s, j}} x}\right)\right) s \\
\quad+\frac{2^{\tau_{-t, j}-1}}{n-2}\left(D f\left(\overline{2^{\tau_{t, j}} x}\right)-D f\left(\overline{-2^{\tau_{t, j} x}}\right)\right) t \| \\
\leq \sum_{j=m}^{m+m^{\prime}-1} \Phi_{j}(x)
\end{gathered}
$$

for all $x_{1}, x_{2}, \ldots, x_{n} \in X \backslash\{0\}$ and all nonnegative integers $m, m^{\prime}$ with $m^{\prime}>0$. From (3), (4), and (14), it follows that the sequence $\left\{J_{m} f(x)\right\}$ is Cauchy for all $x \in X \backslash\{0\}$. Since $Y$ is complete, the sequence $\left\{J_{m} f(x)\right\}$ converges. From this and $\lim _{m \rightarrow \infty} J_{m} f(0)=0$, we can define the mapping $F: X \rightarrow Y$ by

$$
F(x):=\lim _{m \rightarrow \infty} J_{m} f(x)
$$

for all $x \in X$. Moreover, letting $m=0$ and taking the limit as $m^{\prime} \rightarrow \infty$ in (14), we get the inequality (12). Notice that 
$\lim _{m \rightarrow \infty} J_{m} f(0)=0, \lim _{m \rightarrow \infty} 4^{-s m} \varphi\left(2^{s m} x_{1}, \ldots, 2^{s m} x_{n}\right)=$ 0 , and $\lim _{m \rightarrow \infty} 2^{-t m} \varphi\left(2^{t m} x_{1}, \ldots, 2^{t m} x_{n}\right)=0$ for all $x_{1}, x_{2}, \ldots, x_{n} \in X \backslash\{0\}$. Hence, it follows from (11) and the definition of $F$ that

$$
\begin{aligned}
D F\left(x_{1}, x_{2}, \ldots, x_{n}\right) & \\
=\lim _{m \rightarrow \infty} \frac{1}{2}\left(4^{-s m}(\right. & D f\left(2^{s m} x_{1}, \ldots, 2^{s m} x_{n}\right) \\
+ & \left.D f\left(-2^{s m} x_{1}, \ldots,-2^{s m} x_{n}\right)\right) \\
+2^{-t m}( & D f\left(2^{t m} x_{1}, \ldots, 2^{t m} x_{n}\right) \\
& \left.\left.-D f\left(-2^{t m} x_{1}, \ldots,-2^{t m} x_{n}\right)\right)\right)=0
\end{aligned}
$$

for all $x_{1}, x_{2}, \ldots, x_{n} \in X \backslash\{0\}$.

Now, let $F^{\prime}: X \rightarrow Y$ be another mapping satisfying (8) for all $x_{1}, x_{2}, \ldots, x_{n} \in X \backslash\{0\}$ and (12) with $F^{\prime}(0)=0$. Using Lemma $1,(12)$, and $F^{\prime}(0)=0=F(0)$, we obtain

$$
\begin{aligned}
& \left\|F(x)-F^{\prime}(x)\right\| \\
& \leq\left\|J_{m} F(x)-J_{m} F^{\prime}(x)\right\| \\
& \leq \frac{4^{-s m}}{2}\left(\left\|(f-F)\left(2^{s m} x\right)-\frac{n+2}{3 n} f(0)\right\|\right. \\
& +\left\|\left(f-F^{\prime}\right)\left(2^{s m} x\right)-\frac{n+2}{3 n} f(0)\right\| \\
& +\left\|(f-F)\left(-2^{s m} x\right)-\frac{n+2}{3 n} f(0)\right\| \\
& \left.+\left\|\left(f-F^{\prime}\right)\left(-2^{s m} x\right)-\frac{n+2}{3 n} f(0)\right\|\right) \\
& +\frac{2^{-t m}}{2}\left(\left\|(f-F)\left(2^{t m} x\right)-\frac{n+2}{3 n} f(0)\right\|\right. \\
& +\left\|\left(f-F^{\prime}\right)\left(2^{t m} x\right)-\frac{n+2}{3 n} f(0)\right\| \\
& +\left\|(f-F)\left(-2^{t m} x\right)-\frac{n+2}{3 n} f(0)\right\| \\
& \left.+\left\|\left(f-F^{\prime}\right)\left(-2^{t m} x\right)-\frac{n+2}{3 n} f(0)\right\|\right)
\end{aligned}
$$

for all $x \in X \backslash\{0\}$ and all positive integers $m$. It follows from (12) and (17) that

$$
\begin{aligned}
& \left\|F(x)-F^{\prime}(x)\right\| \\
& \quad \leq \sum_{j=0}^{\infty} 2\left(4^{-s m} \Phi_{j}\left(2^{s m} x\right)+2^{-t m} \Phi_{j}\left(2^{t m} x\right)\right)
\end{aligned}
$$

for all $x \in X \backslash\{0\}$ and all positive integers $m$. We can easily show that the terms on the right-hand side of the inequality
(18) tend to 0 as $m \rightarrow \infty$ for the cases $(s, t)=(1,1)$ and $(s, t)=(-1,-1)$. For the case $(s, t)=(1,-1)$, we have

$$
\begin{aligned}
& n(n-2) \sum_{j=0}^{\infty}\left(4^{-m} \Phi_{j}\left(2^{m} x\right)+2^{m} \Phi_{j}\left(2^{-m} x\right)\right) \\
& =\sum_{j=0}^{\infty} \frac{2 \varphi\left(\overline{2^{j+m} x}\right)+2 \varphi\left(\overline{-2^{j+m} x}\right)}{4^{j+m+1}} \\
& +\frac{2^{j} n}{2^{2 m+1}}\left(\sum_{j=0}^{m / 2-1}+\sum_{j=m / 2}^{m-1}+\sum_{j=m}^{\infty}\right) \\
& \times\left(\varphi\left(\overline{\frac{2^{m} x}{2^{j+1}}}\right)+\varphi\left(\overline{\frac{-2^{m} x}{2^{j+1}}}\right)\right) \\
& +\frac{2^{m}}{2^{2 j+1}}\left(\sum_{j=0}^{m / 2-1}+\sum_{j=m / 2}^{m-1}+\sum_{j=m}^{\infty}\right) \\
& \times\left(\varphi\left(\overline{\frac{2^{j} x}{2^{m}}}\right)+\varphi\left(\overline{\frac{-2^{j} x}{2^{m}}}\right)\right) \\
& +\sum_{j=0}^{\infty} \frac{2^{j+m} n}{2}\left(\varphi\left(\overline{2^{-j-m-1} x}\right)+\varphi\left(\overline{-2^{-j-m-1} x}\right)\right) \\
& \leq\left(\frac{1}{2} \sum_{j=m}^{\infty}+n \sum_{j=m / 2}^{m-1}+\frac{n}{2^{m / 2}} \sum_{j=0}^{m / 2-1}\right) \\
& \times\left(\frac{\varphi\left(\overline{2^{j} x}\right)+\varphi\left(\overline{-2^{j} x}\right)}{4^{j}}\right) \\
& +\left(\frac{n}{2^{m}} \sum_{j=1}^{\infty}+\sum_{j=m / 2+1}^{m}+\frac{1}{2^{m / 2}} \sum_{j=1}^{m / 2}\right) \\
& \times\left(2^{j}\left(\varphi\left(\overline{\frac{x}{2^{j}}}\right)+\varphi\left(\overline{\frac{-x}{2^{j}}}\right)\right)\right) \\
& +\frac{1}{2^{m}} \sum_{j=0}^{\infty} \frac{\varphi\left(\overline{2^{j} x}\right)+\varphi\left(\overline{-2^{j} x}\right)}{4^{j}} \\
& +\frac{n}{4} \sum_{j=m+1}^{\infty} 2^{j}\left(\varphi\left(\overline{\frac{x}{2^{j}}}\right)+\varphi\left(\overline{\frac{-x}{2^{j}}}\right)\right)
\end{aligned}
$$

for all $x \in X \backslash\{0\}$ and all positive even integers $m$. So, we also show that the terms on the right-hand side of the inequality (18) tend to 0 as $m \rightarrow \infty$ for the cases $(s, t)=(1,-1)$. Using the equality $F(0)=0=F^{\prime}(0)$, we can conclude that $F(x)=$ $F^{\prime}(x)$ for all $x \in X$. This proves the uniqueness of $F$.

Corollary 3. Let $p \neq 1,2$ be a real number. Suppose that $f$ : $X \rightarrow Y$ is a mapping such that

$$
\left\|D f\left(x_{1}, x_{2}, \ldots, x_{n}\right)\right\| \leq\left\|x_{1}\right\|^{p}+\left\|x_{2}\right\|^{p}+\cdots+\left\|x_{n}\right\|^{p}
$$


for all $x_{1}, x_{2}, \ldots, x_{n} \in X \backslash\{0\}$ (with $f(0)=0$ if $p>2$ ). Then, there exists a unique mapping $F$ satisfying (8) for all $x_{1}, x_{2}, \ldots, x_{n} \in X \backslash\{0\}$ and

$$
\begin{aligned}
& \|f(x)-F(x)\| \\
& \leq\left(\frac{4}{\left|2^{p}-4\right|}+\frac{n}{\left|2^{p}-2\right|}\right) \frac{\|x\|^{p}}{n-2}, \quad \text { if } p>0, \\
& \left\|f(x)-\frac{n+2}{3 n} f(0)-F(x)\right\| \\
& \leq\left(\frac{4}{\left|2^{p}-4\right|}+\frac{n}{\left|2^{p}-2\right|}\right) \frac{1}{n-2}, \quad \text { if } p=0, \\
& f(x)=F(x), \quad \text { if } p<0
\end{aligned}
$$

for all $x \in X \backslash\{0\}$ with $F(0)=0$.

Proof. Put $\varphi\left(x_{1}, x_{2}, \ldots, x_{n}\right)=\left\|x_{1}\right\|^{p}+\left\|x_{2}\right\|^{p}+\cdots+\left\|x_{n}\right\|^{p}$ for all $x_{1}, x_{2}, \ldots, x_{n} \in X \backslash\{0\}$. By Theorem 2 , there exists a unique mapping $F$ satisfying (8) for all $x_{1}, x_{2}, \ldots, x_{n} \in X \backslash\{0\}$ and

$$
\begin{aligned}
& \left\|f(x)-\frac{n+2}{3 n} f(0)-F(x)\right\| \\
& \quad \leq\left(\frac{4}{\left|2^{p}-4\right|}+\frac{n}{\left|2^{p}-2\right|}\right) \frac{\|x\|^{p}}{n-2}
\end{aligned}
$$

for all $x \in X \backslash\{0\}$ with $F(0)=0$. From these, we get the inequalities

$$
\begin{aligned}
& \frac{(n-1)\left(n^{2}-4\right)}{6 n}\|f(0)\| \\
& \leq\|(D f-D F)(k x, k x, \ldots, k x)\| \\
& +\left\|(F-f)(n k x)-\frac{n+2}{3 n} f(0)\right\| \\
& +n(n-2)\left\|(F-f)(k x)-\frac{n+2}{3 n} f(0)\right\| \\
& +\frac{n(n-1)}{2}\left\|(f-F)(2 k x)-\frac{n+2}{3 n} f(0)\right\| \\
& \leq\left(n+\left(n^{p}+n(n-2)+\frac{n(n-1) 2^{p}}{2}\right)\right. \\
& \left.\quad \times\left(\frac{4}{\left|2^{p}-4\right|}+\frac{n}{\left|2^{p}-2\right|}\right)\right) k^{p}\|x\|^{p}
\end{aligned}
$$

for all $x \in X \backslash\{0\}$ and all positive real numbers $k$. Taking the limit as $k \rightarrow \infty$ or $k \rightarrow 0$ in the above inequality, we have $f(0)=0$ if $p \neq 0$. Hence, if $p \neq 0,1,2$, then the inequality

$$
\|f(x)-F(x)\| \leq\left(\frac{4}{\left|2^{p}-4\right|}+\frac{n}{\left|2^{p}-2\right|}\right) \frac{\|x\|^{p}}{n-2}
$$

for all $x \in X \backslash\{0\}$ follows from (23). If $p<0$, then we get the inequalities

$$
\begin{aligned}
& \|f(x)-F(x)\| \\
& \leq \frac{1}{n-1}(\|(D f-D F)((-k+1) x, k x, \ldots, k x)\| \\
& +\|(f-F)(((n-2) k+1) x)\| \\
& +(n-1)(n-2)\|(f-F)(k x)\| \\
& +(n-2)\|(f-F)((-k+1) x)\| \\
& \left.\quad+\frac{(n-1)(n-2)}{2}\|(f-F)(2 k x)\|\right) \\
& \leq\left(k^{p}+\frac{(2 k)^{p}}{2}+\frac{(-k+1)^{p}}{n-1}+\frac{((n-2) k+1)^{p}}{(n-1)(n-2)}\right) \\
& \times\left(\frac{4}{\left|2^{p}-4\right|}+\frac{n}{\left|2^{p}-2\right|}\right)\|x\|^{p} \\
& +\left(\frac{(-k+1)^{p}}{n-1}+k^{p}\right)\|x\|^{p}
\end{aligned}
$$

for all $x \in X \backslash\{0\}$ and all positive integers $k$. Taking the limit as $k \rightarrow \infty$ in the above inequality, we get $F(x)=f(x)$ for all $x \in X \backslash\{0\}$. Since $f(0)=0=F(0)$, the equality $f(x)=F(x)$ holds for all $x \in X$. The result follows from this, (23), and (25).

Lemma 4. If $f: X \rightarrow Y$ is a mapping satisfying (8) for all $x_{1}, x_{2}, \ldots, x_{n} \in X \backslash\{0\}$ with $f(0)=0$ and $f(t x)$ is continuous in $t$ for each fixed $x$, then $f$ is represented by

$$
f(r x)=\left(\frac{f(x)+f(-x)}{2}\right) r^{2}+\left(\frac{f(x)-f(-x)}{2}\right) r
$$

for all $x \in X$ and all $r \in \mathbb{R}$.

Proof. We will prove the equality

$$
f(m x)=\left(\frac{f(x)+f(-x)}{2}\right) m^{2}+\left(\frac{f(x)-f(-x)}{2}\right) m
$$

for all integers $m$. First, we will use the induction on $m$ to prove the equality (28) for all nonnegative integers $m$. Note that $f(0)=0$. We can easily prove it for the cases $m=0,1$. For the case $m=2$, we can show that

$$
\begin{aligned}
f(2 x)= & -\frac{2}{n(n-2)}(D f(\bar{x})+D f(\overline{-x})) \\
& -\frac{1}{2(n-2)}(D f(\bar{x})-D f(\overline{-x}))+3 f(x)+f(-x) \\
= & \left(\frac{f(x)+f(-x)}{2}\right) 2^{2}+\left(\frac{f(x)-f(-x)}{2}\right) 2
\end{aligned}
$$


for all $x \in X$. Assume that (28) holds for all $x \in X$ and all nonnegative integers $k(\leq m)$. Then, we obtain

$$
\begin{aligned}
f(( & +1) x) \\
= & -D f\left(m x, \hat{x}_{x, \ldots, x,-x, \ldots,-x}^{n / 2}\right)+2 f(m x) \\
& -f((m-1) x)-\frac{n-4}{4} f(-2 x)-\frac{n}{4} f(2 x) \\
& +n f(x)+(n-2) f(-x) \\
= & \left(2 m^{2}-(m-1)^{2}-\frac{n-4}{4} \cdot(-2)^{2}-\frac{n}{4} \cdot 2^{2}\right. \\
& \left.+n \cdot 1^{2}+(n-2) \cdot(-1)^{2}\right) \\
& \times\left(\frac{f(x)+f(-x)}{2}\right)+\left(\frac{f(x)-f(-x)}{2}\right) \\
& \times\left(2 m-(m-1)-\frac{n-4}{4} \cdot(-2)\right. \\
= & \left.\quad \frac{f(x)+f(-x)}{2}\right)(m+1)^{2} \\
& +\left(\frac{f(x)-f(-x)}{2}\right)(m+1)
\end{aligned}
$$

which completes (28) for all nonnegative integers $m$. Using the similar method, we also can prove the equality (28) for all negative integers $m$. By (28), we get the equalities

$$
\begin{gathered}
\frac{f(m x)+f(-m x)}{2}=\left(\frac{f(x)+f(-x)}{2}\right) m^{2}, \\
\frac{f(m x)-f(-m x)}{2}=\left(\frac{f(x)-f(-x)}{2}\right) m, \\
\frac{f(x / m)+f(-x / m)}{2}=\left(\frac{f(x)+f(-x)}{2}\right) \frac{1}{m^{2}}, \\
\frac{f(x / m)-f(-x / m)}{2}=\frac{f(x)-f(-x)}{2 m}
\end{gathered}
$$

for all $x \in X$ and all integers $m \neq 0$. Hence,

$$
\begin{aligned}
f\left(\frac{p}{q} x\right) & \\
= & \frac{f((p / q) x)+f(-(p / q) x)}{2} \\
& +\frac{f((p / q) x)-f(-(p / q) x)}{2}
\end{aligned}
$$

$$
\begin{aligned}
= & \left(\frac{f(x / q)+f(-x / q)}{2}\right) p^{2} \\
& +\left(\frac{f(x / q)-f(-x / q)}{2}\right) p \\
= & \left(\frac{f(x)+f(-x)}{2}\right) \frac{p^{2}}{q^{2}}+\left(\frac{f(x)-f(-x)}{2}\right) \frac{p}{q}
\end{aligned}
$$

for all $x \in X$ and all integers $p, q(\neq 0)$. If $r \in \mathbb{R}$, then there exists a rational sequence $\left\{r_{m}\right\}$ satisfying $\lim _{m \rightarrow \infty} r_{m}=r$. Since $f(t x)$ is continuous in $t$ for each fixed $x$, we have

$$
\begin{aligned}
f(r x) & =\lim _{m \rightarrow \infty} f\left(r_{m} x\right) \\
& =\lim _{m \rightarrow \infty}\left(\frac{f(x)+f(-x)}{2}\right) r_{m}^{2}+\left(\frac{f(x)-f(-x)}{2}\right) r_{m} \\
& =\left(\frac{f(x)+f(-x)}{2}\right) r^{2}+\left(\frac{f(x)-f(-x)}{2}\right) r
\end{aligned}
$$

for all $x \in X$.

\section{Stability of the Functional Equation (2) ( $n$ Is Odd)}

Let $(s, t), \varphi, D f\left(x_{1}, x_{2}, \ldots, x_{n}\right)$, and $\tau_{k, m}$ be as in Section 2 . In this section, let $n$ be an odd integer greater than 2 . For convenience, we use the following abbreviations in this section for a given mapping $f: X \rightarrow Y$ :

$$
\begin{aligned}
& J_{m} f(x)=\frac{1}{2}\left(4 ^ { - s m } \left(f\left(2^{s m} x\right)+f\left(-2^{s m} x\right)\right.\right. \\
& \left.-\frac{2(n+1)}{3(n-1)} f(0)\right) \\
& \left.+2^{-t m}\left(f\left(2^{t m} x\right)-f\left(-2^{t m} x\right)\right)\right) \text {, } \\
& \bar{x}=(\overbrace{x, \ldots, x}^{(n+1) / 2}, \frac{(n-1) / 2}{-x, \ldots,-x}),
\end{aligned}
$$

for $x \in X$. From these, we get

$$
\begin{array}{rl}
J_{m} & f(x)-J_{m+1} f(x) \\
= & \frac{4^{\tau_{-s, m}}+1}{2(n-1)(n-1)}\left(D f\left(\overline{2^{\tau_{s, m} x}}\right)+D f\left(\overline{-2^{\tau_{s, m} x}}\right)\right) s \\
& +\frac{2^{\tau_{-t, m}}}{n-1}\left(D f\left(\overline{2^{\tau_{t, m}} x}\right)-D f\left(\overline{-2^{\tau_{t, m}} x}\right)\right) t
\end{array}
$$

for all $x \in X$.

Using (35) and a similar method in the proof of Lemma 1, we get the following lemma. 
Lemma 5. If $f: X \rightarrow Y$ is a mapping satisfying (8) for all $x_{1}, x_{2}, \ldots, x_{n} \in X \backslash\{0\}$, then

$$
J_{m} f(x)=f(x)-\frac{n+1}{3(n-1)} f(0)
$$

for all $x \in X \backslash\{0\}$.

From (35), Lemma 5, and similar methods used in Theorem 2, we get the following theorem.

Theorem 6. If $f: X \rightarrow Y$ is a unique mapping satisfying (11) for all $x_{1}, x_{2}, \ldots, x_{n} \in X \backslash\{0\}$ with $\lim _{m \rightarrow \infty} J_{m} f(0)=0$, then there exists a unique mapping $F: X \rightarrow Y$ satisfying (8) for all $x_{1}, x_{2}, \ldots, x_{n} \in X \backslash\{0\}$ and

$$
\left\|f(x)-\frac{n+1}{3(n-1)} f(0)-F(x)\right\| \leq \sum_{j=0}^{\infty} \Phi_{j}(x)
$$

for all $x \in X \backslash\{0\}$ with $F(0)=0$, where $\Phi_{j}$ are the mappings defined by

$$
\begin{aligned}
\Phi_{j}(x):= & \frac{4^{\tau_{-s, m}+1}}{2(n-1)(n-1)}\left(\varphi\left(\overline{2^{\tau_{s, m} x}}\right)+\varphi\left(\overline{-2^{\tau_{s, m} x}}\right)\right) \\
& +\frac{2^{\tau_{-t, m}}}{n-1}\left(\varphi\left(\overline{2^{\tau_{t, m}} x}\right)+\varphi\left(\overline{-2^{\tau_{t, m}} x}\right)\right)
\end{aligned}
$$

for all $x \in X \backslash\{0\}$.

From Theorem 6 and similar methods used in Corollary 3, we get the following corollary.

Corollary 7. Let $p \neq 1,2$ be a real number. Suppose that $f$ : $X \rightarrow Y$ is a mapping satisfying (20) for all $x_{1}, x_{2}, \ldots, x_{n} \in$ $X \backslash\{0\}$ (with $f(0)=0$ if $p>2$ ). Then, there exists a unique mapping $F$ satisfying (8) for all $x_{1}, x_{2}, \ldots, x_{n} \in X \backslash\{0\}$ and

$$
\begin{aligned}
& \|f(x)-F(x)\| \\
& \leq\left(\frac{4}{(n-1)\left|2^{p}-4\right|}+\frac{2}{\left|2^{p}-2\right|}\right) \frac{n\|x\|^{p}}{n-1}, \\
& \text { if } p>0, \\
& \left\|f(x)-\frac{n+1}{3(n-1)} f(0)-F(x)\right\| \\
& \leq\left(\frac{4}{(n-1)\left|2^{p}-4\right|}+\frac{2}{\left|2^{p}-2\right|}\right) \frac{n}{n-1}, \\
& \quad \text { if } p=0,
\end{aligned}
$$

for all $x \in X \backslash\{0\}$ with $F(0)=0$.
Proof. Put $\varphi\left(x_{1}, x_{2}, \ldots, x_{n}\right)=\left\|x_{1}\right\|^{p}+\left\|x_{2}\right\|^{p}+\cdots+\left\|x_{n}\right\|^{p}$ for all $x_{1}, x_{2}, \ldots, x_{n} \in X \backslash\{0\}$. By Theorem 6 , there exists a unique mapping $F$ satisfying (8) for all $x_{1}, x_{2}, \ldots, x_{n} \in X \backslash\{0\}$ and

$$
\begin{aligned}
& \left\|f(x)-\frac{n+1}{3(n-1)} f(0)-F(x)\right\| \\
& \quad \leq\left(\frac{4}{(n-1)\left|2^{p}-4\right|}+\frac{2}{\left|2^{p}-2\right|}\right) \frac{n\|x\|^{p}}{n-1}
\end{aligned}
$$

for all $x \in X \backslash\{0\}$ with $F(0)=0$. From these, we get the inequalities

$$
\begin{aligned}
& \frac{(n+1)(n-2)}{6}\|f(0)\| \\
& \leq\left\|(F-f)(n k x)-\frac{n+1}{3(n-1)} f(0)\right\| \\
& \quad+n(n-2)\left\|(F-f)(k x)-\frac{n+1}{3(n-1)} f(0)\right\| \\
& \quad+\frac{n(n-1)}{2}\left\|(f-F)(2 k x)-\frac{n+1}{3(n-1)} f(0)\right\| \\
& \quad+\|(D f-D F)(k x, k x, \ldots, k x)\| \\
& \leq \\
& \quad\left(\left(n^{p}+n(n-2)+\frac{n(n-1) 2^{p}}{2}\right)\right. \\
& \left.\quad \times\left(\frac{4}{(n-1)\left|2^{p}-4\right|}+\frac{2}{\left|2^{p}-2\right|}\right) \frac{n}{n-1}+n\right) k^{p}\|x\|^{p}
\end{aligned}
$$

for all $x \in X \backslash\{0\}$ and all positive real numbers $k$. Taking the limit as $k \rightarrow \infty$ or $k \rightarrow 0$ in the above inequality, we have $f(0)=0$ if $p \neq 0$. Hence, if $p \neq 0,1,2$, then the inequality

$$
\|f(x)-F(x)\| \leq\left(\frac{4}{(n-1)\left|2^{p}-4\right|}+\frac{2}{\left|2^{p}-2\right|}\right) \frac{n\|x\|^{p}}{n-1}
$$

for all $x \in X \backslash\{0\}$ follows from (40). If $p<0$, then we get the inequalities

$$
\begin{aligned}
& \|f(x)-F(x)\| \\
& \leq \frac{1}{n-1}(\|(D f-D F)((-k+1) x, k x, \ldots, k x)\| \\
& + \\
& +(n-F)(((n-2) k+1) x) \| \\
& +(n-2)\|(f-F)((-k+1) x)\| \\
& \left.+\frac{(n-1)(n-2)}{2}\|(f-F)(2 k x)\|\right)
\end{aligned}
$$




$$
\begin{gathered}
\leq(n-2)\left(k^{p}+\frac{(2 k)^{p}}{2}+\frac{(-k+1)^{p}}{n-1}\right. \\
\left.+\frac{((n-2) k+1)^{p}}{(n-1)(n-2)}\right) \\
\times\left(\frac{4}{\left|2^{p}-4\right|(n-1)}+\frac{2}{\left|2^{p}-2\right|}\right) \frac{n\|x\|^{p}}{n-1} \\
+\left(\frac{(-k+1)^{p}}{n-1}+k^{p}\right)\|x\|^{p}
\end{gathered}
$$

for all $x \in X \backslash\{0\}$ and all positive integers $k$. Taking the limit as $k \rightarrow \infty$ in the above inequality, we get $F(x)=f(x)$ for all $x \in X \backslash\{0\}$. Since $f(0)=0=F(0)$, the equality $f(x)=F(x)$ holds for all $x \in X$. The result follows from this, (40), and (42).

From similar methods used in Lemma 4, we get the following lemma.

Lemma 8. If $f: X \rightarrow Y$ is a mapping satisfying (8) for all $x_{1}, x_{2}, \ldots, x_{n} \in X \backslash\{0\}$ with $f(0)=0$ and $f(t x)$ is continuous in $t$ for each fixed $x$, then $f$ is represented by

$$
f(r x)=\left(\frac{f(x)+f(-x)}{2}\right) r^{2}+\left(\frac{f(x)-f(-x)}{2}\right) r
$$

for all $x \in X$ and all $r \in \mathbb{R}$.

Proof. We will use the induction on $m$ to prove (44) for all nonnegative integers $m$. Note that $f(0)=0$. We can easily prove it for the cases $m=0,1$. For the case $m=2$, we can show that

$$
\begin{aligned}
f(2 x)= & -\frac{2}{(n-1)^{2}}(D f(\bar{x})+D f(\overline{-x})) \\
& -\frac{1}{n-1}(D f(\bar{x})-D f(\overline{-x}))+3 f(x)+f(-x) \\
= & \left(\frac{f(x)+f(-x)}{2}\right) 2^{2}+\left(\frac{f(x)-f(-x)}{2}\right) 2
\end{aligned}
$$

for all $x \in X$. Assume that (44) holds for all $x \in X$ and all nonnegative integers $k(\leq m)$. Then, we obtain

$$
\begin{aligned}
& f((m+1) x) \\
& =-\frac{2}{n-1} D f(m x, \overbrace{x, \ldots, x}^{(n-1) / 2}, \frac{(n-1) / 2}{-x, \ldots,-x})+2 f(m x) \\
& \quad-f((m-1) x)-\frac{n-3}{4} f(-2 x)-\frac{n-3}{4} f(2 x) \\
& \quad+(n-2) f(x)+(n-2) f(-x)
\end{aligned}
$$

$$
\begin{aligned}
= & \left(2 m^{2}-(m-1)^{2}-\frac{n-3}{4} \cdot\left((-2)^{2}+2^{2}\right)\right. \\
& \left.+(n-2)\left(1+(-1)^{2}\right)\right) \\
& \times\left(\frac{f(x)+f(-x)}{2}\right)+\left(\frac{f(x)-f(-x)}{2}\right) \\
& \times\left(2 m-(m-1)-\frac{n-3}{4}\right. \\
= & \left.\quad \frac{f(x)+f(-x)}{2}\right)(m+1)^{2}+\left(\frac{f(x)-f(-x)}{2}\right)(m+1)
\end{aligned}
$$

which completes the proof of (44). The remainder of the proof is the same in the proof of Lemma 4.

Corollary 9. If $f: X \rightarrow Y$ is a mapping satisfying (8) for all $x_{1}, x_{2}, \ldots, x_{n} \in X \backslash\{0\}$, then $f(0)=0$.

Proof. Put $p=-1$. Then, we have

$$
\left\|D f\left(x_{1}, x_{2}, \ldots, x_{n}\right)\right\|=0 \leq\left\|x_{1}\right\|^{p}+\left\|x_{2}\right\|^{p}+\cdots+\left\|x_{n}\right\|^{p}
$$

for all $x_{1}, x_{2}, \ldots, x_{n} \in X \backslash\{0\}$. By Corollaries 3 and $7, f(x)=$ $F(x)$ for all $x \in X$ with $F(0)=0$. So, we get the desired result.

Corollary 10. Let $p<0$ be a real number and $n>2$ an integer. Suppose that $f: \mathbb{R} \rightarrow \mathbb{R}$ is a mapping satisfying

$$
\left|D f\left(x_{1}, x_{2}, \ldots, x_{n}\right)\right| \leq\left|x_{1}\right|^{p}+\left|x_{2}\right|^{p}+\cdots+\left|x_{n}\right|^{p}
$$

for all $x_{1}, x_{2}, \ldots, x_{n} \in \mathbb{R} \backslash\{0\}$ and $f$ is continuous. Then, $f$ is represented by

$$
f(x)=\left(\frac{f(1)+f(-1)}{2}\right) x^{2}+\frac{f(1)-f(-1)}{2} x
$$

for all $x \in \mathbb{R}$ and $\operatorname{Df}\left(x_{1}, x_{2}, \ldots, x_{n}\right)=0$ for all $x_{1}, x_{2}, \ldots, x_{n} \in$ $\mathbb{R}$.

Proof. If $n$ is even, then the equality (49) follows from Corollary 3 and Lemma 4 . If $n$ is odd, then the equality (49) follows from Corollary 7 and Lemma 8. And we can easily show that the function defined by (49) satisfies the functional equation $D f\left(x_{1}, x_{2}, \ldots, x_{n}\right)=0$ for all $x_{1}, x_{2}, \ldots, x_{n} \in$ $\mathbb{R}$.

\section{Another Proof for the Stability of the Functional Equation (2)}

Let $(s, t), D f\left(x_{1}, x_{2}, \ldots, x_{n}\right), \tau_{k, m}$ be as in Section 2. In this section, Let $n$ be a fixed integer greater than 2 and let $\varphi$ : $X^{n} \rightarrow[0, \infty)$ be a function satisfying the conditions (3) and (4) for all $x_{1}, x_{2}, \ldots, x_{n} \in X$. For convenience, we use 
the following abbreviations in this section for a given mapping $f: X \rightarrow Y$ :

$$
\begin{gathered}
J_{m} f(x)=\frac{1}{2}\left(4 ^ { - s m } \left(f\left(2^{s m} x\right)+f\left(-2^{s m} x\right)\right.\right. \\
\left.+\frac{(n-4)(n+1)}{3} f(0)\right) \\
\left.+2^{-t m}\left(f\left(2^{t m} x\right)-f\left(-2^{t m} x\right)\right)\right), \\
\bar{x}=(x,-x, x, 0, \ldots, 0),
\end{gathered}
$$

for all $x \in X$. From these, we get

$$
\begin{aligned}
J_{m} f(x)-J_{m+1} f(x) \\
=\frac{4^{\tau_{-s, m}}}{2}\left(D f\left(\overline{2^{\tau_{s, m} x}}\right)+D f\left(\overline{-2^{\tau_{s, m} x}}\right)\right) s \\
\quad+\frac{2^{\tau_{-t, m}}}{2}\left(D f\left(\overline{2^{\tau_{t, m}} x}\right)-D f\left(\overline{-2^{\tau_{t, m}} x}\right)\right) t
\end{aligned}
$$

for all $x \in X$. Using (51) and a similar method in the proof of Lemma 1 , we get the following lemma.

Lemma 11. If $f: X \rightarrow Y$ is a quadratic-additive mapping, then

$$
J_{m} f(x)=f(x)
$$

for all $x \in X$.

Theorem 12 (compare with Theorem 3.1 in [15]). Suppose that $f: X \rightarrow Y$ is a mapping such that

$$
\left\|D f\left(x_{1}, x_{2}, \ldots, x_{n}\right)\right\| \leq \varphi\left(x_{1}, x_{2}, \ldots, x_{n}\right)
$$

for all $x_{1}, x_{2}, \ldots, x_{n} \in X$. Then, there exists a unique quadratic-additive mapping $F: X \rightarrow Y$ such that

$$
\left\|f(x)+\frac{(n-4)(n+1)}{6} f(0)-F(x)\right\| \leq \sum_{j=0}^{\infty} \Phi_{j}(x)
$$

for all $x \in X$, where $\Phi_{j}$ are the mappings defined by

$$
\begin{aligned}
\Phi_{j}(x):= & \frac{4^{\tau_{-s, j}}}{2}\left(\varphi\left(\overline{2^{\tau_{s, j}} x}\right)+\varphi\left(\overline{-2^{\tau_{s, j} x}}\right)\right) \\
& +\frac{2^{\tau_{-t, j}}}{2}\left(\varphi\left(\overline{2^{\tau_{t, j}} x}\right)+\varphi\left(\overline{-2^{\tau_{t, j}} x}\right)\right)
\end{aligned}
$$

for all $x \in X$.

Proof. Note that

$$
\begin{aligned}
\left\|J_{m} f(0)\right\| & =\frac{4^{-s m}(n-1)(n-2)}{6}\|f(0)\| \\
& =\frac{4^{-s m}}{3}\|D f(0,0, \ldots, 0)\| \\
& \leq \frac{4^{-s m}}{3} \varphi(0,0, \ldots, 0)
\end{aligned}
$$

for all positive integers $m$. It follows from (3) that $\lim _{m \rightarrow \infty} J_{m} f(0)=0$. From this, (51), Lemma 11, and similar methods used in Theorem 2, we obtain this theorem.

Corollary 13 (compare with Corollary 3.3 in [15]). Let $p \neq 1,2$ be a positive real number. Suppose that $f: X \rightarrow Y$ is a mapping such that

$$
\left\|D f\left(x_{1}, x_{2}, \ldots, x_{n}\right)\right\| \leq\left\|x_{1}\right\|^{p}+\left\|x_{2}\right\|^{p}+\cdots+\left\|x_{n}\right\|^{p}
$$

for all $x_{1}, x_{2}, \ldots, x_{n} \in X$. Then, there exists a unique quadratic-additive mapping $F$ such that

$$
\|f(x)-F(x)\| \leq\left(\frac{3}{\left|2^{p}-4\right|}+\frac{3}{\left|2^{p}-2\right|}\right)\|x\|^{p}
$$

for all $x \in X$.

Proof. Since $\|f(0)\|=(2 /(n-1)(n-2))\|D f(0,0, \ldots, 0)\| \leq 0$, we get $f(0)=0$. From Theorem 12 and similar methods used in Corollary 3, we obtain this corollary.

From Theorem 12 and similar methods used in Corollary 3 , we get the following corollary.

Corollary 14 (compare with Corollary 3.2 in [15]). Suppose that $f: X \rightarrow Y$ is a mapping such that

$$
\left\|D f\left(x_{1}, x_{2}, \ldots, x_{n}\right)\right\| \leq \varepsilon
$$

for all $x_{1}, x_{2}, \ldots, x_{n} \in X$. Then, there exists a unique quadratic-additive mapping $F$ such that

$$
\left\|f(x)+\frac{(n-4)(n+1)}{6} f(0)-F(x)\right\| \leq \frac{4 \varepsilon}{3}
$$

for all $x \in X$.

\section{Conflict of Interests}

The author declares that there is no conflict of interests regarding the publication of this paper.

\section{Acknowledgments}

This research was supported by Basic Science Research Program through the National Research Foundation of Korea (NRF) funded by the Ministry of Education, Science and Technology (no. 2012R1A1A4A01002971).

\section{References}

[1] S. M. Ulam, A Collection of Mathematical Problems, Interscience, New York, NY, USA, 1960.

[2] D. H. Hyers, "On the stability of the linear functional equation," Proceedings of the National Academy of Sciences of the United States of America, vol. 27, pp. 222-224, 1941.

[3] T. Aoki, "On the stability of the linear transformation in Banach spaces," Journal of the Mathematical Society of Japan, vol. 2, pp. 64-66, 1950. 
[4] T. M. Rassias, "On the stability of the linear mapping in Banach spaces," Proceedings of the American Mathematical Society, vol. 72, no. 2, pp. 297-300, 1978.

[5] P. Găvruţa, "A generalization of the Hyers-Ulam-Rassias stability of approximately additive mappings," Journal of Mathematical Analysis and Applications, vol. 184, no. 3, pp. 431-436, 1994.

[6] G. H. Kim, "On the stability of functional equations with square-symmetric operation," Mathematical Inequalities \& Applications, vol. 4, no. 2, pp. 257-266, 2001.

[7] Y.-H. Lee, "On the stability of the monomial functional equation," Bulletin of the Korean Mathematical Society, vol. 45, no. 2, pp. 397-403, 2008.

[8] Y.-H. Lee and K.-W. Jun, "A generalization of the Hyers-UlamRassias stability of Jensen's equation," Journal of Mathematical Analysis and Applications, vol. 238, no. 1, pp. 305-315, 1999.

[9] Y.-H. Lee and K.-W. Jun, "On the stability of approximately additive mappings," Proceedings of the American Mathematical Society, vol. 128, no. 5, pp. 1361-1369, 2000.

[10] K.-W. Jun and H.-M. Kim, "On the stability of an $n$-dimensional quadratic and additive functional equation," Mathematical Inequalities \& Applications, vol. 9, no. 1, pp. 153-165, 2006.

[11] M. Janfada and R. Shourvazi, "Solutions and the generalized Hyers-Ulam-Rassias stability of a generalized quadraticadditive functional equation," Abstract and Applied Analysis, vol. 2011, Article ID 326951, 19 pages, 2011.

[12] S. S. Jin and Y.-H. Lee, "Fuzzy stability of an $n$-dimensional quadratic and additive type functional equation," International Journal of Mathematical Analysis, vol. 7, no. 29-32, pp. 1513-1530, 2013.

[13] S. S. Jin and Y.-H. Lee, "A fixed point approach to the stability of the $n$-dimensional quadratic and additive functional equation," International Journal of Mathematical Analysis, vol. 7, no. 29-32, pp. 1557-1573, 2013.

[14] S. S. Jin and Y.-H. Lee, "On the stability of the $n$-dimensional quadratic and additive functional equation in random normed spaces via fixed point method," International Journal of Mathematical Analysis, vol. 7, no. 49-52, pp. 2413-2428, 2013.

[15] P. Nakmahachalasint, "On the generalized Ulam-GavrutaRassias stability of mixed-type linear and Euler-LagrangeRassias functional equations," International Journal of Mathematics and Mathematical Sciences, vol. 2007, Article ID 63239, 10 pages, 2007.

[16] Y.-H. Lee, "On the quadratic additive type functional equations," International Journal of Mathematical Analysis, vol. 7, no. 37-40, pp. 1935-1948, 2013.

[17] S.-M. Jung, "On the Hyers-Ulam stability of the functional equations that have the quadratic property," Journal of Mathematical Analysis and Applications, vol. 222, no. 1, pp. 126-137, 1998.

[18] S. S. Jin and Y.-H. Lee, "Fuzzy stability of a mixed type functional equation," Journal of Inequalities and Applications, vol. 2011, p. 70, 2011.

[19] S. S. Jin and Y.-H. Lee, "A fixed point approach to the stability of the mixed type functional equation," Honam Mathematical Journal, vol. 34, no. 1, pp. 19-34, 2012.

[20] S. S. Jin and Y.-H. Lee, "On the stability of the mixed type functional equation in random normed spaces via fixed point method," Journal of the Korean Society of Mathematical Education. Series B. The Pure and Applied Mathematics, vol. 19, no. 1, pp. 59-71, 2012.
[21] I.-S. Chang, E. H. Lee, and H.-M. Kim, "On Hyers-UlamRassias stability of a quadratic functional equation," Mathematical Inequalities \& Applications, vol. 6, no. 1, pp. 87-95, 2003.

[22] S. S. Jin and Y.-H. Lee, "A fixed point approach to the stability of the quadratic-additive functional equation," Journal of the Korea Society of Mathematical Education. Series B. The Pure and Applied Mathematics, vol. 18, no. 4, pp. 313-328, 2011.

[23] S. S. Jin and Y. H. Lee, "Fuzzy stability of a quadratic-additive functional equation," International Journal of Mathematics and Mathematical Sciences, vol. 2011, Article ID 504802, 16 pages, 2011.

[24] S. S. Jin and Y. H. Lee, "On the stability of the quadratic-additive functional equation in random normed spaces via fixed point method," Journal of the Chungcheong Mathematical Society, vol. 25, pp. 201-215, 2012.

[25] Y. W. Lee, "Stability of a generalized quadratic functional equation with Jensen type," Bulletin of the Korean Mathematical Society, vol. 42, no. 1, pp. 57-73, 2005. 


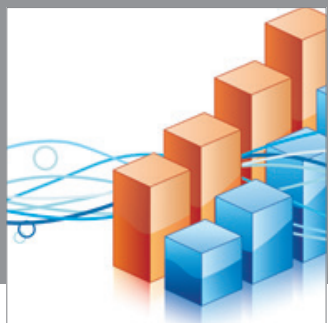

Advances in

Operations Research

mansans

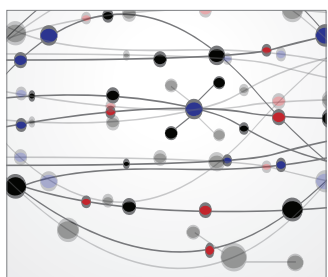

The Scientific World Journal
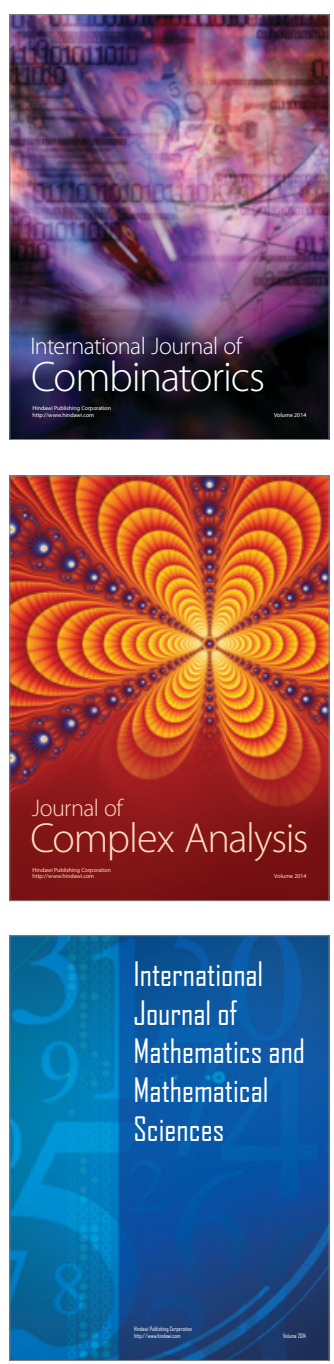
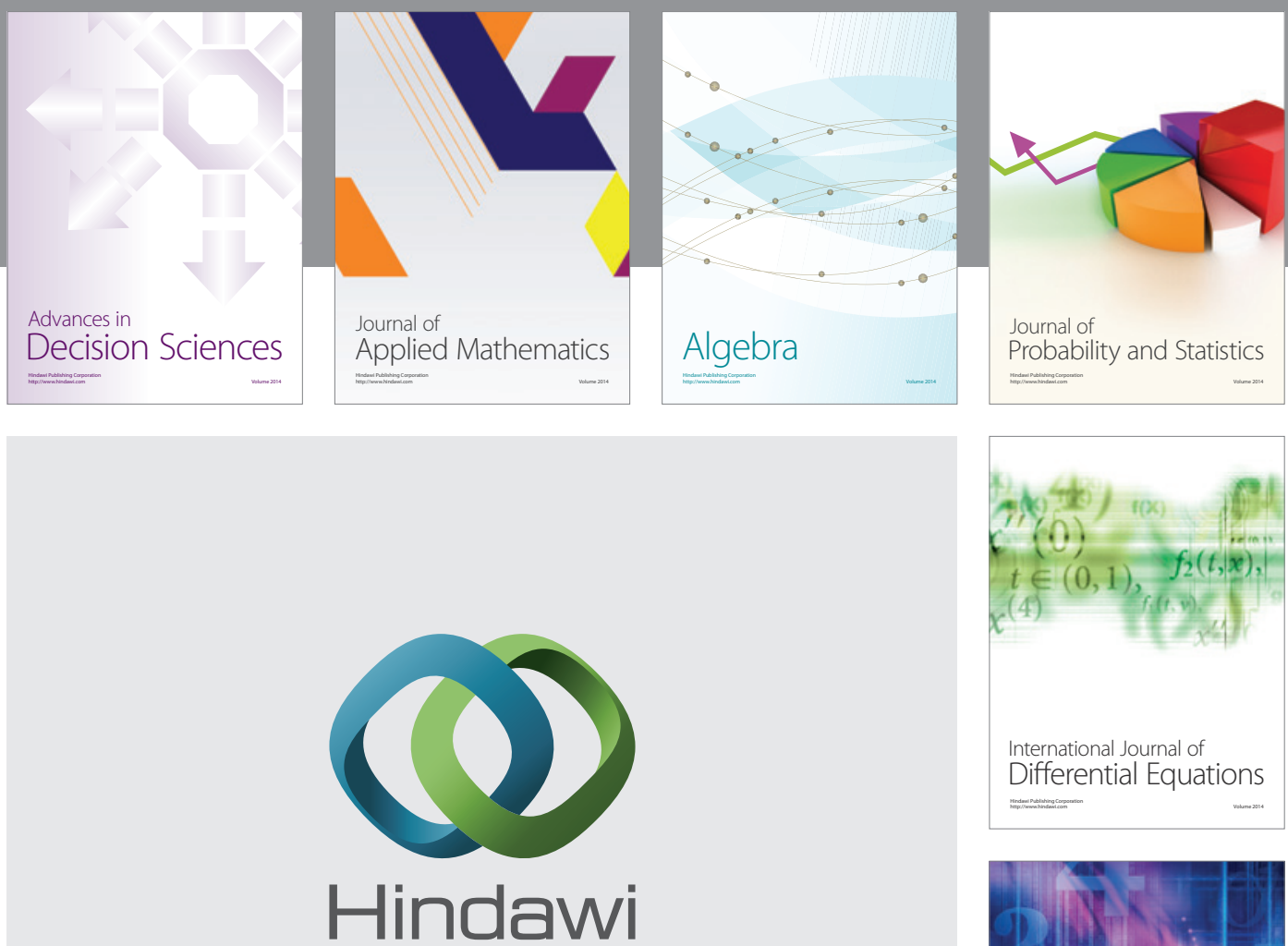

Submit your manuscripts at http://www.hindawi.com
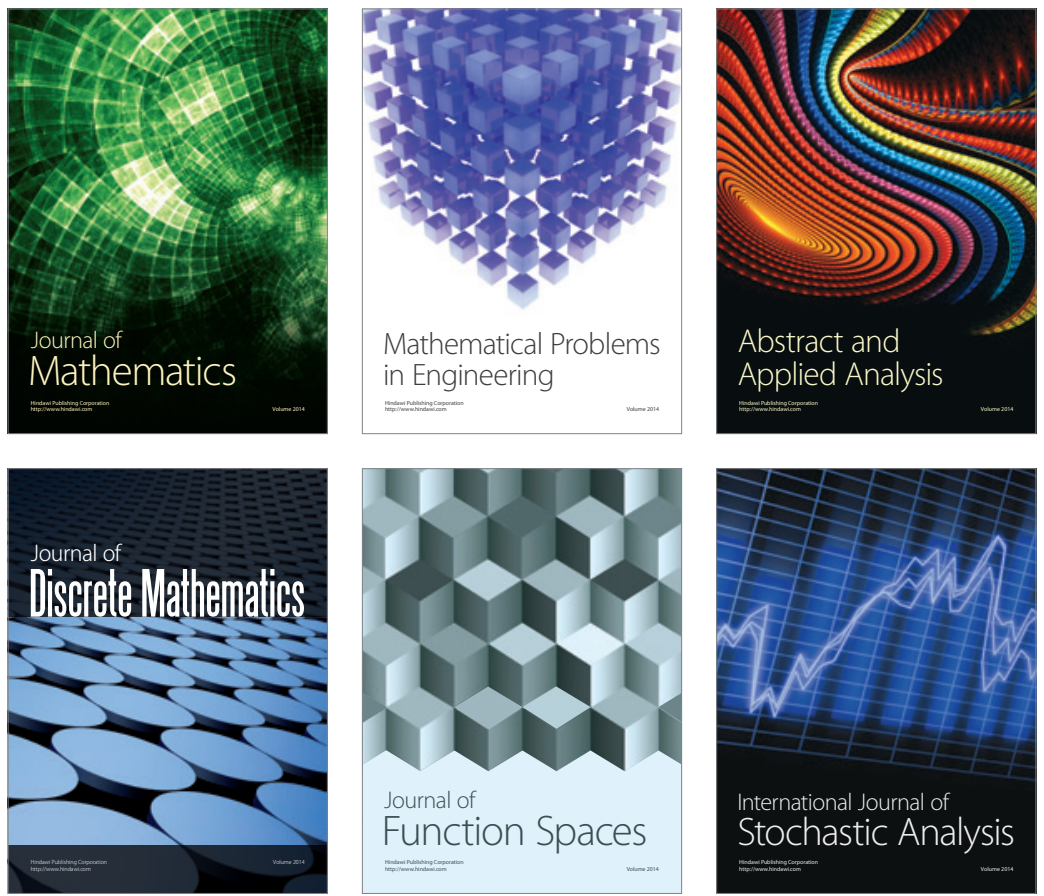

Journal of

Function Spaces

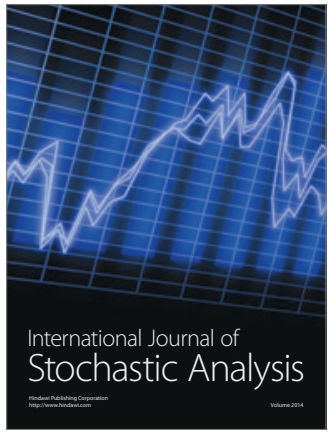

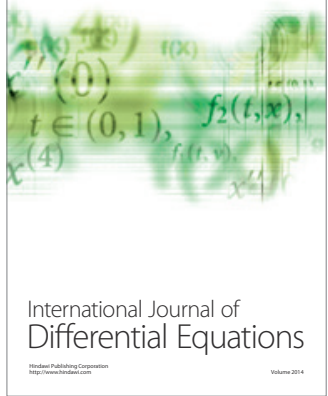
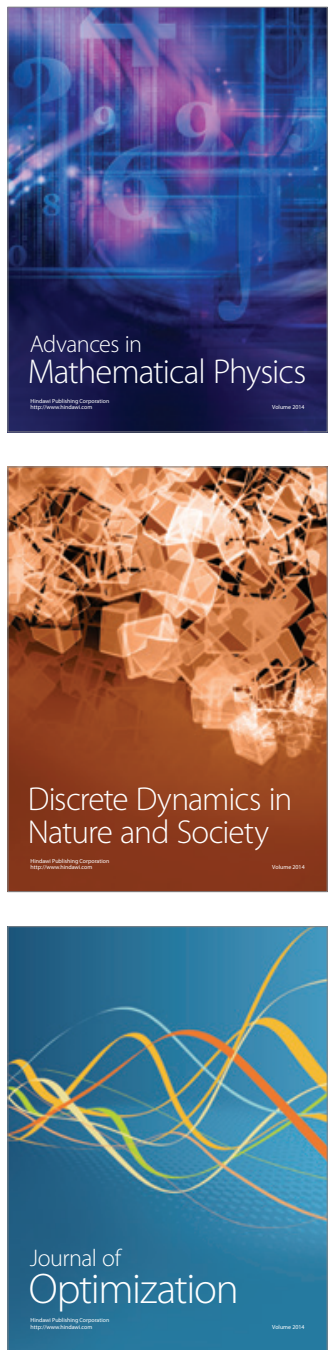